\title{
A PSICOLOGIA E OS PSICÓLOGOS(AS) NOS ESTUDOS EM EDUCAÇÃO SEXUAL: ENCAMINHAMENTOS PARA PESQUISA
}

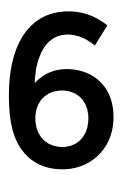

\section{PSYCHOLOGY AND PSYCHOLOGISTS IN STUDIES IN SEXUAL EDUCATION: REFERENCES FOR RESEARCH}

MONTEIRO, SOLANGE APARECIDA DE SOUZA

DOUTORANDA EM EDUCAÇÃO ESCOLAR DA UNESP/ARARAQUARA SOLMONTEIRO@IFSP.EDU.BR

ORCID ID: HTTPS://ORCID.ORG/0000-0002-1640-0266

SILVA, CLAUDIONOR RENATO DA

DOUTOR EM EDUCAÇÃO ESCOLAR PELA UNESP/ARARAQUARA PROFESSOR ADJUNTO NA UNIVERSIDADE FEDERAL DE GOIÁS

CLAUDIONORSIL@GMAIL.COM

ORCID ID: HTTPS://ORCID.ORG/0000-0003-1693-4804

\section{RESUMO}

A problemática deste estudo é elencar encaminhamentos à formação em pesquisa para psicólogos(as) e, respectivas contribuições à Educação (à Pedagogia, em especial), no tocante a produção stricto das ciências psicológicas nos temas da Educação Sexual. Objetivo geral é apontar a produção de conhecimento em Educação Sexual de psicólogos e psicólogas na área da Psicologia (programas de pós-graduação brasileiros). De metodologia bibliográfica a partir de levantamentos no Catálogo de Teses e Dissertações da CAPES, acompanhado de buscas em outras fontes online como a plataforma lattes (currículo lattes dos pesquisadores/as) e consultas à plataforma Sucupira para localização dos conceitos dos Programas em Psicologia, elaboram-se três encaminhamentos à pesquisa em Psicologia para os estudos da Educação Sexual e três contribuições dadas à própria área e à área da Educação e Educação Sexual no incentivo e na formação de novos(as) pesquisadores(as), a partir da Iniciação Científica.

Palavras-chave: psicologia; educação sexual; pesquisa. 


\section{ABSTRACT}

The problem of this study is to list referrals to research training for psychologists and their respective contributions to Education (to Pedagogy, in particular), regarding the strict production of psychological sciences in the themes of Sexual Education. General objective is to point out the production of knowledge in Sexual Education of psychologists and psychologists in the area of Psychology (Brazilian graduate programs). Bibliographic methodology based on surveys in the CAPES Thesis and Dissertations Catalog, accompanied by searches in other online sources such as the lattes platform (researchers' lattes curriculum) and consultations with the Sucupira platform to locate the concepts of the Psychology Programs, three guidelines for research in Psychology are elaborated for the studies of Sexual Education and three contributions given to the area itself and to the area of Education and Sexual Education in encouraging and training new researchers, starting with Scientific Initiation.

Keywords: psychology; sex education; research.

\section{INTRODUÇÃO}

A Psicologia é uma das muitas áreas em que a Educação Sexual tem obtido evidência nos temas advindos da pessoa humana, seja em relação saúde, seja em relação à educação e, também, relativos aos âmbitos sociais.

No interesse de "adentrar" nessa área importante das ciências humanas, a Psicologia, no que diz respeito, exclusivamente, às suas contribuições para a Educação Sexual, os autores se juntaram para colocar em questão esta presença da Psicologia na Educação Sexual e a produção de psicólogos(as), a partir dos programas de pós-graduação em Psicologia no Brasil.

É objetivo da pesquisa encontrar nas produções em Psicologia, produzidas por psicólogos(as), alguns apontamentos que encaminhem pesquisas em Educação Sexual, com incentivos para a pesquisa, desde a Iniciação Científica até a pós-graduação, estimulando a formação de novos(as) pesquisadores nos cursos de formação de psicólogos, mas também, em áreas de interface, como a Educação, em particular, a Pedagogia e, por conseguinte, para outras licenciaturas, tendo como finalidade a produção em pesquisa na área da Educação Sexual. 
O problema que instiga os autores nessa proposta textual: o que a área da Psicologia e os psicólogos(as) têm produzido na área da Educação Sexual e quais suas contribuições com encaminhamentos à formação e produção de pesquisa e pesquisadores? Quais as temáticas da área com possíveis contribuições para outras áreas, como a Educação, em particular, a Educação Sexual?

Objetiva-se, então, apontar a Psicologia como importante área de contributo à Educação Sexual, uma contribuição para além da área, trata-se de contribuições à formação de psicólogos(as) e também de educadores(as) sexuais, com explícitas contribuições ao currículo da educação escolar, bem como os espaços outros externos a esse e tão importantes quanto.

A metodologia é o da Pesquisa Bibliográfica procurando atender à pergunta da problemática levantada, conforme Sampieri; Collado, Lucio (2006). A metodologia permite atender ao problema da pesquisa; na orientação dada pelos levantamentos realizados, permite, também, pontuar nesse universo, a presença da Psicologia e dos psicólogos(as) na área da Educação Sexual.

Os procedimentos de acesso aos documentos e informações contidas nos sites dos Programas de Pós-Graduação em Psicologia, bem como, dados sobre as notas dos conceitos destes Programas na CAPES, bem como, dados dos autores(as), sobretudo, sua formação em psicologia, complementam os dados que são construídos a partir da indicação de Programas de Pós-graduação, com Linhas que se definam como propulsoras do tema da Educação Sexual, consultados no Catálogo de Dissertações e Teses da Capes, em que se identificam os Programas e também os trabalhos produzidos (dissertações e teses), a partir da leitura de seus resumos, que determinam, de alguma maneira, as tendências de estudos na área específica da Psicologia que possam ter, ou não, contribuições à área da Educação Sexual.

Uma das conclusões importantes da presente pesquisa, iniciada pelos autores e compartilhada nesse dossiê, são os encaminhamentos à pesquisa em Educação Sexual, da psicologia e dos psicólogos(as). Ampliam-se as conclusões na motivação para encaminhamentos de investigações para a formação em Educação Sexual, desde a Iniciação Científica estendendo-se para a pós-graduação stricto sensu, em programas de Psicologia e programas de Educação, em particular, programas de Educação Sexual. 
A primeira seção organiza-se com nossas considerações sobre alguns aspectos da pesquisa em Psicologia e dos fins destas pesquisas na área da Educação Sexual, seus temas e focos de estudo, produzidas por psicólogos(as). Como afirma Foucault (1999, p.16), "[...] levar em consideração o fato de se falar de sexo, quem fala, os lugares e os pontos de vista de que se fala, as instituições que incitam a fazê-lo, que armazenam e difundem o que dele se diz [...] a "colocação do sexo em discurso".

Assume-se nesta pesquisa que a Psicologia é um "lugar" que nos "incita", nos motiva e nos faz "intelectualizar" o falar em sexualidade humana; "fazer" Educação Sexual, "fazer" Pesquisa, em Psicologia, na Educação Sexual. Podemos, sim, pensar que além do campo educacional, estamos nos discursos e instâncias da saúde, mas, por hora, focalizaremos a Psicologia nas Ciências Humanas, muito embora, os resultados apresentam várias interfaces em saúde.

Segundo a Organização Mundial da Saúde (OMS) a terminologia "saúde" está relacionada ao "estado de bem-estar físico, mental e social", ou seja, ao falar de saúde devemos ampliar e referenciar um conjunto de condições coletivas que permitem a continuidade da própria sociedade, em produzir e reproduzir-se de maneira saudável. Nesse aspecto, o psicólogo(a) como profissional de saúde deve utilizar seu conhecimento de psicologia no intuito do aumento da qualidade de vida da sociedade; o psicólogo(a) deve desenvolver seu trabalho através da aplicação das técnicas da psicologia, em diversos contextos (consultórios, escolas, instituições, produção acadêmica, entre outros); contextos em ações que visam a promoção da saúde e considera as condições objetivas e subjetivas da vida de cada pessoa, compreendendo sua singularidade.

Os profissionais da psicologia se dedicam às pesquisas ligadas às ciências psicológicas, enquanto corpo de conhecimento que resulta em produções acadêmicas e transmissão de conhecimentos dos resultados encontrados. Essas atuações dão sustentabilidade para a execução da profissão. (BOCK, FURTADO; TEIXEIRA, 2001).

\section{A PESQUISA EM PSICOLOGIA E A PESQUISA EM EDUCAÇÃO SEXUAL NA PSICOLOGIA}

O surgimento da Psicologia, enquanto área é quase que, concomitante, ao seu surgimento como ato ou "campo" de pesquisa. O primeiro a impulsionar o "paralelo" deste surgimento da área e da 
prática de pesquisa foi Renê Descartes, mas só se consolida nos estudos do comportamento humano ou quando se começou a pensar o humano na escala do não laboratorial, com Wilhelm Wundt - retomaremos sobre esse "início", mais adiante.

Bock, Furtado; Teixeira (2001) afirmam que a psicologia moderna, nascida na Alemanha, no final do século XIX, através de Wundt, Fechner e Weber, angariou pesquisadores e estudiosos que passaram a produzir o conhecimento através de novos padrões; padrões de produções, numa postura metodológica, com proposta teórica em pesquisa.

Surgem, a partir de então, as primeiras abordagens em Psicologia. Dentre as diversas abordagens surge Sigmund Freud como um divisor de águas nos estudos sobre a sexualidade nas interfaces Saúde, Medicina e Psicologia. Com a psicanálise, tem-se a constituição do que se pode dizer, a principal abordagem para a sexualidade infantil, com a tese de que a maioria dos desejos reprimidos e pensamentos advêm de conflitos de ordem sexual.

Kupfer (2007) observou em seus estudos sobre as teorias de Freud que no interior da própria sexualidade existia/havia o desprazer advindo da moral e, ligava a doença nervosa nesta mesma moralidade; a cada aprofundamento a que Freud avançava em seus estudos sobre sexualidade foi entendendo a necessidade de formação de uma prática à Educação para o desenvolvimento psíquico saudável do indivíduo.

Algumas áreas tratam sobre a sexualidade humana em diferentes contextos sejam sobre a história da evolução cultural, sobre a fisiologia, aspectos anatômicos, psicológicos; a sexologia busca aglutinar todas as áreas e respectivos estudos, que envolvam a sexualidade humana.

A pesquisa e a produção científica se fazem importantes para construção contemporânea de métodos e ferramentas que englobam as necessidades da realidade atual, visando auxiliar os profissionais da área da psicologia no colaborar com a saúde mental dos indivíduos, de modo geral, de acordo com a competência da psicologia, nas múltiplas facetas da sexualidade; deve elucidar qual a diferença entre sexo e sexualidade/ prazer; o sentido(s) que está ligado aos conteúdos da sexualidade, ao prazer.

Alguns autores(as) nos auxiliam na construção deste estudo, não só no histórico da ciência da Psicologia e sua produção, mas, auxiliam também na construção de elementos da pesquisa desta ciência, na particularidade do tema da Educação Sexual e suas temáticas, a saber: 
Katz (1954), Marx; Hillix (1963), Keller (1970), Barroso; Broschini; (1982; 1985), Figueiredo (1991), Minayo (1994), Camino; Pereira (2000), Rey (2005), Borges et al. (2013) e Antunes (2014).

A pesquisa em Psicologia nos temas da Educação Sexual necessita de alguns "explicitamentos", como por exemplo, a compreensão do que estamos a considerar como Educação Sexual.

Partimos de uma concepção foucaultiana para a Educação Sexual, para este texto, uma concepção pautada na, [...] necessidade reconhecida de que é preciso [...]. Deve-se falar de sexo, e falar publicamente [...] exige procedimentos de gestão; deve ser assumido por discursos analíticos. (FOUCAULT, 1999, p. 27).

Entende-se, assim, que a Educação Sexual encontra na Psicologia, o lugar de seu discurso(s) e, ao mesmo tempo, a Psicologia permite-se ser a gestora dos discursos sobre sexo, sexualidade, gênero, etc., num exercício de análise rigoroso, tal como deve ser a produção em ciência, a produção em Educação Sexual.

Estamos a considerar pesquisas da área da Psicologia, na Educação Sexual, aquelas investigações ou produções de conhecimento que tenham como base a definição de Educação Sexual, desenvolvida a partir dos anos 1980 e que vem se reconstruindo na contemporaneidade; a base em referenciais como Werebe, Suplicy, Figueiró, Paulo Rennes, Ana Claudia Maia, Claudia Ribeiro, Sônia Melo e tantos outros(as). Outra base: a presença de temas da Educação Sexual, como: gênero, sexualidade, psicanálise na sexualidade infantil, feminilidades, masculinidades, sexualidade de pessoas com deficiências, movimentos sociais como LGBT, Queers, etc. Portanto, relações de interface não apenas educacional, mas também de saúde e de dimensões sociais e políticas, mais amplas.

Desta forma ao afirmarmos o que seria e o que se "enquadraria" como uma pesquisa em psicologia no(s) tema(s) da Educação Sexual, a compreensão construída é de uma pesquisa na área da psicologia, produzida por graduados em psicologia, orientados(as) por psicólogos(as), todos(as), interessados(as) em investigações nos temas de estudos da Educação Sexual, na abrangência de todos os seus temas; focados nos processos de ensino intencionais (escolares) e não intencionais (mídias, por exemplo, e aqueles processos familiares e "individualizados) e que estejam no âmago da formação humana, desenvolvimento humano.

Barroso; Broschini (1982) apontam que até os anos 1970, o conhecimento da temática da sexualidade já era vasto, porém, pouco 
articulado. Os discursos deste período, são retomadas, em pleno século XXI e evidenciam preconceitos e discriminações; preconceitos e discriminações homofóbicos, de resistência às atividades de professores(as); há falta de diálogos sobre e em diversidade; há uma inexistente capacidade de uma troca honesta de conhecimentos e Direitos, com ênfase nas diferenças existenciais, fantasias, dúvidas e receios relativos ao sexo e à sexualidade. $\mathrm{Na}$ atualidade esse "vasto" conhecimento ainda é velado em discursos sobre a "família de bem" que coloca o sexo e a sexualidade num mesmo discurso "vitoriano" em que sexo e sexualidade é tema que não deve ser falado publicamente, apenas no "secreto" e na família, jamais na escola. O que é contraditório, pois o sexo e (nossa) sexualidade está no corpo(s), está nas pessoas, está em todos os lugares, nos filmes, livros, ruas e bares, etc. Neste sentido, a Psicologia e a Educação Sexual, recorrem ao conjunto da obra de Michel Foucault, para ampliação das fontes das ciências da psicologia em tratar a sexualidade humana na subjetividade dos indivíduos para o bem da coletividade.

A psicologia desde os tempos primórdios de seu campo de estudo, com ciência, tem o conhecimento que as questões sexuais interligadas aos aspectos morais é fonte de angústia. Para a psicanálise é a busca pelo prazer que dá espaço ao impulso sexual, chamada libido. A maturação faz parte do desenvolvimento da libido que tem início desde os primeiros contatos com o mundo até a puberdade.

A criança de forma progressiva engatinha, se levanta, anda, ou seja, antes de investir a libido no outro é necessário aprender o que é o prazer.

Faz parte da maturação, o prazer oral (conhecer o mundo através da boca, mamar, lamber objetos, experimentar); prazer anal (expulsão e/ou retenção das fezes); prazer fálico (prazer por manipular órgãos genitais).

Dessa forma, com o crescimento da criança, o impulso sexual vai se delineando, aos cinco anos, período em que a criança já possui uma sexualidade razoavelmente definida.

Após essa idade até a puberdade, a criança passa uma fase adaptativa denominada pela psicanálise de latência, quando se abandonará o objeto sexual das relações parentais para escolhas fora da família.

Devemos pontuar também o complexo de Édipo que ocorre através atração sexual da criança, não no sentido genital; criança gosta de carinho 
e cobrará carinho (filho/mãe e filha/pai). Mediante essa maturação gradativa é possível aferir que a sexualidade surge desde muito cedo e que as manifestações primárias não são genitais e, sim, a organização do impulso da libido que, posteriormente, será primordial na busca do prazer sexual. É denominado a todo esse processo maturacional, como sexualidade, algo muito mais abrangente que sexo de forma "rasa".

Para "fora" da psicanálise há várias abordagens das ciências da psicologia que se debruçaram sobre a sexualidade humana. Em seu conjunto, formam um arcabouço de produção riquíssimo para a Educação Sexual.

Para subsidiar a reflexão sobre a pesquisa em Psicologia, nos temas da Educação Sexual, mas, também, para responder a pergunta da pesquisa, se efetua o levantamento no Catálogo de Teses da CAPES, como detalhado, a seguir, nos procedimentos sob a Pesquisa Bibliográfica em Sampieri; Collado; Lúcio (2006).

\section{DADOS DE FONTES BIBLIOGRÁFICAS - ELEMENTOS ADICIONAIS PARA OS ENCAMINHAMENTOS DE PESQUISA EM EDUCAÇÃO SEXUAL NA ÁREA DA PSICOLOGIA}

Esta seção apresenta uma síntese do levantamento bibliográfico realizado (Sampieri; Collado, Lucio, 2006) e adicionam-se "caminhos" para levantamento de novas outras informações que consolidem as respostas à problemática de pesquisa, por exemplo, buscas nos sites dos programas de pós-graduação em Psicologia; buscas no currículo lattes dos pesquisadores(as), graduados(as) em Psicologia.

Saturar os dados é um desafio ao trabalho do pesquisador(a) bibliográfico, no intuito de explicitar seus argumentos e justificar suas análises na resposta ao problema levantado, o que permite, ademais, como afirmam, Sampieri; Collado, Lucio (2006) construírem-se novos e novas temáticas de pesquisas e até encaminhamentos de propostas de definições, conceitos e indicar elementos para teorização e teorias no campo estudado. E, é desta forma, que o presente texto se estrutura a partir desta seção, para concluir com encaminhamentos em pesquisas nas ciências da psicologia e contribuições à Educação Sexul - em específico, para licenciaturas, por exemplo, a Pedagogia.

No Catálogo de Teses e Dissertações da CAPES, a partir do descritor composto "psicologia; educação sexual" são encontrados 1.439 resultados (trabalhos, pesquisas), sendo 1.184 dissertações e 255 
teses, no período de 2014 a 2019. O levantamento foi realizado nos primeiros dias de setembro de 2020.

A Grande Área do Conhecimento é as Ciências Humanas; Área do Conhecimento, Área de Avaliação e nome do Programa estão todos sob a Psicologia, interesse desta investigação.

As cinco primeiras Universidades que mais produzem trabalhos de dissertações e teses no Brasil são apresentadas no Quadro 1.

QUADRO 1 - Os cinco programas de pós-graduação em Psicologia que mais produzem sob os temas da Educação Sexual: dissertações e teses. Período: 2014 a 2019

\begin{tabular}{|l|c|c|c|}
\hline Local/Conceito CAPES & N. $^{\text {o }}$ Trabalhos & Dissertações & Teses \\
\hline $\begin{array}{l}\text { Universidade Federal do } \\
\text { Rio Grande do Sul (UFR- } \\
\text { GS) }\end{array}$ & 159 & 80 & 79 \\
Conceito 7 & & & \\
\hline $\begin{array}{l}\text { Universidade Federal } \\
\text { do Rio Grande do Norte } \\
\text { (UFRN) }\end{array}$ & 147 & 110 & 37 \\
Conceito 5 & & & \\
\hline $\begin{array}{l}\text { Universidade Federal do } \\
\text { Espírito Santo (UFES) } \\
\text { Conceito 5 }\end{array}$ & 137 & 89 & 48 \\
\hline $\begin{array}{l}\text { Universidade Federal de } \\
\text { Júz de Fora (UFJF) } \\
\text { Conceito 5 }\end{array}$ & 133 & 107 & 26 \\
\hline $\begin{array}{l}\text { Pontíficia Universidade } \\
\text { Católica de Goiás (PUC- } \\
\text {-GOIÁS) } \\
\text { Conceito 4 }\end{array}$ & 118 & & 35 \\
\hline
\end{tabular}

Fonte: Elaborado pelos autores.

Juntas, as cinco universidades com seus Programas de PósGraduação em Psicologia, produzem um pouco mais de $45 \%$ de toda a produção levantada na pesquisa bibliográfica: do total dos 1.439 
trabalhos, as cinco primeiras, produziram, no período, 694 dissertações e teses.

Cada uma destas universidades representa uma região do Brasil (sul, nordeste, sudeste e centro-oeste), exceto o norte. No elencamento, como representando esta região brasileira, mas não com representativa produção, dentre as cinco universidades elencadas, destacam-se os trabalhos de Pantoja (2019) da Universidade Federal do Pará, no Programa de Mestrado em Psicologia (Conceito Capes 4) e a dissertação de Miwa (2019), defendida na Universidade Federal do Amazonas, no Programa de Pós-Graduação em Psicologia, de Conceito CAPES 3.

Do total das produções destas cinco universidades e seus Programas em Psicologia (694 trabalhos - nível um) se fez um segundo nível de recorte bibliográfico com até 100 trabalhos, distribuídos entre as cinco universidades para uma leitura dos seus resumos na íntegra. Destes 100, recorta-se um terceiro nível, apenas para alguns apontamentos para fins deste artigo e são comentados a seguir; permitem uma síntese bem próxima ao real do objeto de investigação que gera a problemática desta pesquisa.

É preciso reiterar, em relação aos procedimentos, seguindo as proposições de Sampieri; Collado, Lucio (2006) que, para além do levantamento das das produções destes Programas de Pós-Graduação em Psicologias, se fez uma busca nos sites destes Programas, com o intuito de identificar, nas Áreas de Concentração, nas Linhas, nas Ementas das Disciplinas - quando necessário - o "lugar" da terminologia "Educação Sexual", num primeiro momento, e, num momento posterior, as terminologias que se "enquadram" nas pesquisas e teorias da Educação Sexual, como gênero, como sexualidade, etc.; termos mais abrangentes como diversidade sexual, direitos humanos, feminismo, feminilidades, masculinidades, etc.

Em todas as cinco universidades, em seus respectivos Programas, não apresentam termos como "educação sexual", "gênero" ou sexualidade. Terminologias muito recorrentes e comuns, quando se investigam propostas na área da Educação Sexual, em particular.

Contudo, se percebe a presença da área da Educação Sexual e seus temas de investigação, mas sob um viés das ciências da psicologia como apresentam os referenciais já discutidos neste artigo.

Na UFRGS, a Linha de Pesquisa em Desenvolvimento Humano traz a seguinte ementa: 
[...] Abrange distintos contextos (família, escola, casal, clínica, trabalho, universidade), aspectos culturais, vulnerabilidades sociais e métodos de avaliação e de intervenção [...]. Também investiga as influências educativas (família, escola, universidade) sobre o sujeito em desenvolvimento. (Site do Programa de Programa de Pós-Graduação em Psicologia da UFRGS).

Na disciplina sob o mesmo nome da Linha de Pesquisa há o compromisso de abordar "[...] diversos domínios do desenvolvimento humano (emocional, da cognição social, moral, da carreira, da família) $[\ldots]^{\prime \prime}$.

Na UFRN, na Linha "Subjetividade, Produção do Cuidado Humanizado em Saúde e Tanatologia", aparece o termo "masculinidades", "diversidade sexual" e outros termos ligados diretamente aos estudos da Educação Sexual. Um dos temas de estudo, a destacar: "[...] a)Organização do cuidado e das práticas em saúde, com foco nas juventudes, masculinidades, diversidade sexual, famílias, vulnerabilidades à infecção pelo HIV/AIDS [...]".

O site da UFRN apresenta temas de pesquisa em andamento. Aparece o termo gênero na temática de pesquisa "Relações de Gênero, território e modos de vida em contextos de pobreza", pertencente à Linha "Políticas Públicas, movimentos sociais e prática do psicólogo", bem como na temática "Psicologia e contextos rurais", em que se investigam as relações de gênero nos movimentos sociais do campo.

Todos os sites dos programas apresentam os trabalhos defendidos, o que é uma importante fonte para estudos bibliográficos na Iniciação Científica, sobretudo.

A UFES com seu Programa de Pós-Graduação em Psicologia, apresenta, na Linha 1 (Processos Psicossociais) a inserção dos termos "gênero", "preconceito", "violência":

A linha de pesquisa Processos Psicossociais abrange o estudo dos processos psicossociais relacionados à identidade, aos processos de comunicação, ao gênero, às relações familiares e conjugais, ao relacionamento interpessoal, ao preconceito, à violência e suas interconexões com contextos culturais. (Site do Programa de Programa de PósGraduação da UFES, grifos nossos). 
Chama a atenção o enfoque dos estudos e pesquisas do Programa de Pós-Graduação da UFJF na Linha "Desenvolvimento Humano e Processos Sócio-Educativos". O objetivo dos estudos são investigações sobre as,

[...] as múltiplas variáveis associadas ao desenvolvimento humano ao longo do curso da vida, sejam elas cognitivas, afetivas, sociais e/ou biológicas. Esse campo da Psicologia está intrinsecamente relacionado aos processos socioeducativos. Assim, ele influencia e é influenciado pelos processos formativos. Neste sentido, essa linha de pesquisa estuda o desenvolvimento e sua relação de dependência e contiguidade com processos educacionais formais e informais. Ao estudar cientificamente as múltiplas variáveis que são afetadas e afetam o desenvolvimento humano, constroem-se conhecimentos que possibilitam planejar e implementar intervenções mais eficazes que levam a um desenvolvimento harmônico do indivíduo ao longo de todo o curso de vida. (Site do Programa de Programa de Pós-Graduação em Psicologia da UFJF).

Destaque na UFJF para a disciplina "Processos psicossociais e cultura do corpo", com a Ementa que traz a seguinte informação:

Estudo das teorias sobre o corpo e sua relação com a cultura contemporânea. Utilização dessas teorias para a compreensão das várias manifestações culturais. Estudo de temas [...] sobre corpo e diversidade. Estudo sobre as teorias biopsicossociais que discutem o corpo em suas diversas dimensões. Estudo sobre a relação entre corpo e estigma sócio-cultural em diferentes dimensões. (Site do Programa de Programa de Pós-Graduação em Psicologia da UFJF).

Dentre as várias temáticas da disciplina está o estudo sobre o corpo em relação à deficiência e a sexualidade humana, a partir do referencial de Assumpção Júnior; Almeida (2008).

A Linha de Pesquisa 2 "Psicologia Social, do Trabalho e das Organizações" na PUC-GOIÁS, destaca, seu objetivo e foco na 
[...] produção de conhecimento sobre fenômenos sociais complexos, abordados a partir do reconhecimento das diferentes modalidades do pensamento social. Organizase em duas vertentes: 1) o estudo das relações entre o indivíduo e a sociedade, e dos processos intra e intergrupais característicos da dinâmica destas relações, [...]. Dentre os vários temas de investigação, encontram-se: [,,,] (4) identidade social; 5) discriminação e preconceito social; [,,1] 7) violência e dialética da exclusão/inclusão social; 8) impacto da ideologia e do contexto sócio-histórico na subjetividade do indivíduo; 9) cultura e o processo de individuação; 10) a construção de sentidos e significados em processos psicossociais de violência contra mulheres, crianças e adolescentes; 12) atendimento psicoterapêutico em redes de atenção a mulheres, crianças e adolescentes; [...]. (Site do Programa de Programa de Pós-Graduação em Psicologia da PUC-GOIÁS).

Uma vez demonstrado o teor dos objetivos dos Programas de PósGraduação em Psicologia, das cinco universidades que mais produzem em Educação Sexual, comenta-se, brevemente, a partir de agora, pelos espaços limitados deste artigo, neste dossiê, o terceiro nível de recorte de dados, elencando os trabalhos da Psicologia com os temas em e na Educação Sexual, produzidos por psicólogos(as) e que encaminham as respostas ainda provisórias desta pesquisa, diante da problemática desenvolvida que coloca em evidência as contribuições da Psicologia e dos psicólogos(as), nos estudos em Educação Sexual.

A tese de Bordini (2014), graduada em psicologia, pela UFRGS, tem como palavras-chave de seu trabalho os termos "gênero" e "sexualidade". Com referencial metodológico na psicologia discursiva (Potter; Wetherell, 1997), a pesquisa se debruça no universo universitário para "sondar" as interações entre homens e mulheres na diversidade sexual.

Angelo Brandelli Costa, graduado em psicologia, pela UFRGS, defendeu sua tese sobre a vulnerabilidade para o HIV em mulheres trans (COSTA, 2015).

Neste estudo de três frentes, Costa (2015) enfatiza a problemática dos testes em psicologia para se determinar sexualidades - o CPS (Escala de Personalidade de Comrey); aponta a vulnerabilidades das mulheres 
trans, em relação à contaminação pelo HIV/Aids e, por último, nas questões sobre a saúde, o preconceito e as dificuldades de acesso das mulheres trans nos serviços públicos.

Estudos sobre a violência, temática fundamental na Educação Sexual, têm acepções/particularidades bem definidas na área da Psicologia. E tal diversidade de violência, ou, violências são observações um pouco mal interpretadas na área da Educação Sexual, o que implica uma maior aproximação da Psicologia no desenvolvimento deste tema no currículo e na formação de professores(as).

Souza (2014) graduada em psicologia pela Universidade de Fortaleza (UNIFOR), estudou a violência sexual intrafamiliar contra as crianças e adolescentes. Sua maior contribuição à Psicologia foi a apresentação do Estado da Arte deste tema na produção científica em Psicologia .

Além da especificidade do estudo de Souza (2014), Hohendorff (2016), graduado em psicologia pelas Faculdades Integradas de Taquara, FACCAT, estudou a violência contra meninos na UFRGS; esta temática é pouco evidenciada pela mídia e também nos estudos da Educação Sexual. É preciso mais estudos sob esse enfoque, para fins de teorização e metodologias, em Psicologia e que possam ser transpostas às pesquisas sobre gênero, geralmente, restritas ao tema da mulher e das meninas, no tocante à violência e abuso sexuais.

Rêgo (2016), formada em Psicologia, pela Universidade Potiguar $($ UnP), trata da violência doméstica em um estudo com mulheres abrigadas, realizado na Universidade Federal do Rio Grande do Norte. Na pesquisa é utilizado a Hermenêutica de Gadamer (Gadamer, 2002; 2012; 2015), para quem, a linguagem é o centro da experiência do sujeito na alteridade; se valoriza a escuta atenta para além da superação do trauma e a retomada, ainda que difícil, de uma certa "normalidade" desejável.

A violência sexual contra adolescentes é um tema trazido pela psicóloga Liane Martinho (Martinho, 2016), formada em graduação na PUC-Goiás. O destaque desse trabalho é a relação que faz com outras violências, traduzindo o entendimento, também no interior da Educação Sexual, das particularidades ou individualidades das "violências".

Ernesto Alvarado Portalino é formado em Psicologia pela Universidad Nacional Mayor de San Marcos (UNMSM) no Peru. Portalino (2017) aborda a questão do preconceito contra a diversidade de gênero entre universitários peruanos. Utiliza a Escala de Preconceito contra 
Diversidade Sexual e de Gênero-Revisada (COSTA, BANDEIRA; NARDI, 2015; COSTA et. al. , 2016).

O resultado do estudo de Portalino (2017) promovem motivações aos novos(as) pesquisadores(as) em Psicologia e também em Educação Sexual, quatno ao juntar abordagens quantitativas (matemática estatística) e qualitativas nos temas em sexualidade humana, assim como nas investigações de Pereira, Monteiro, Camino (2009) que trabalhou também com Escala em seu estudo sobre homossexualidade; acrescentam-se no levantamento sobre o uso de Escalas, Baumel (2014) e Baptista (2017).

Melina Séfora Souza Rebouças é graduada em Psicologia pela Universidade Federal do Rio Grande do Norte. Seu estudo de doutorado (Rebouças, 2015) discorreu sobre o aborto em mulheres em situação de abortamento.

O aborto tem retornado com muita intensidade nos debates nos meios sociais e midiáticos e toda a questão religiosa envolvente. Considera-se, que tal temática seja relevante aos trabalhos de novos(as) pesquisadores na Educação Sexual, sob o viés da Psicologia.

"Investigando o papel da masturbação na sexualidade da mulher" (Baumel 2014), Sergio Werner Baumel que é formado em medicina, pela Universidade de São Paulo (USP) e, em psicologia, pela Universidade Federal do Espírito Santo (UFES), traz em destaque um tema importante para os estudos da feminilidade na Educação Sexual.

O estudo de Baumel (2014) é realizado com estudantes homens e mulheres, universitários(as) da UFES; utiliza-se, dentre outras fontes de dados, da Escala do Índice de Função Sexual Feminina (Rosen et al., 2000) - FSFI (Female Sexual Function Index) -, da Escala Mosher Abreviada de Culpa Sexual (Abramson; Mosher, 1975; Janda; Bazemore, 2011), da Escala Marlowe-Crowne de Desejabilidade Social (Evans, 1982; Gouveia et al., 2009) e da Escala de Satisfação Sexual para Mulheres, na referência e Catão et al. (2010).

Um tema importante para a Educação Sexual é a transsexualidade (Ekins; King, 2006), estudos travestis e transfobia. Neste sentido, é de grande contribuição o trabalho do psicólogo Gustavo Tassis Baptista, formado pela Faculdade Brasileira UNIVIX. Seu Trabalho de Conclusão de Curso, em Psicologia, teve como tema: "Cidadania Travesti? Estereótipos e preconceitos na busca de um lugar além da pista", defendido em 2014.

$\mathrm{Na}$ dissertação de mestrado, no interior do Programa de PósGraduação em Psicologia da UFES, Baptista (2017) traz o referencial 
da Psicologia Positiva, que possui como base os temas bem-estar, a resiliência e o apoio social. Da base referencial, cita a Escala de Bemestar Subjetivo (EBES) nas referências de Albuquerque; Tróccoli (2004) e Pureza et al. (2012).

Odacyr Roberth Moura da Silva é graduado em Psicologia pela Universidade Vale do Rio Doce (UNIVALE) e em Pedagogia, pela UNIUBE (Universidade de Uberaba). Defendeu sua dissertação na Universidade Federal do Espírito Santo (UFES).

Sua dissertação é sobre a identidade sexual, na especificidade de homossexuais masculinos que alegaram terem mudado para sexualidades heteronormativas e todos os desafios aí advindos.

Mozine (2017) é formada em Psicologia pela Universidade Vila Velha (UVV). Em sua dissertação na Universidade Federal do Espírito Santo, a UFES, trata do abuso sexual com destaque aos Conselhos Tutelares. Utiliza-se dos referenciais da Representação Social na Psicologia.

A psicóloga Anna Rita Simião (Simião, 2015), formada em Psicologia pela Universidade Federal de Juíz de Fora, desenvolveu sua dissertação no tema da sexualidade e perversão em Krafft-Ebing. Um trabalho que traz a fonte psicanalítica para os estudos em Educação Sexual. Há vários títulos no levantamento realizado que indicam a forte presença da psicanálise, como se tratou nas seções anteriores. E, em muitas das Linhas estudas, entre as cinco universidades com programas de Psicologia, se viu a presença e o enfoque da psicanálise. Para a área da Educação Sexual isso é muito importante ser aprofundado seja para gênero, sexualidade, violência, angústia, etc.

Glaucia David (David, 2017) é psicóloga formada pelo Centro de Ensino Superior (CES); seu estudo de dissertação na Universidade Federal de Juíz de Fora (UFJF) é sobre o sujeito soropositivo e como são suas vivências nos relacionamentos afetivo-amorosos, depois da descoberta da sua situação em saúde.

Artur Vandré Pitanga (Pitanga, 2016), psicólogo formado pela PUC-Goiás desenvolve sua tese, nesta mesma universidade, concluída em 2016, com o tema dos sentimentos sexuais na relação terapêutica, com base nos trabalhos do psiquiatra Aaron Beck e da psicóloga Marsha R. Linehan.

O Quadro 2 apresenta as palavras-chaves mais recorrentes no terceiro nível de recorte bibliográfico efetuado na pesquisa. Estes dados auxiliam os futuros(as) pesquisadores(as) a desenvolverem suas pesquisas 
na área da Educação Sexual, a partir do contributos da Psicologia e dos psicólogos(as).

QUADRO 2 - Palavras-chave e respectivos referenciais da Psicologia ou produções de psicólogos(as)

\begin{tabular}{|c|c|}
\hline Palavras-Chave & $\begin{array}{c}\text { Pesquisadores(as) psicólogos(as) } \\
\text { e respectivos referenciais de } \\
\text { trabalhos }\end{array}$ \\
\hline Gênero e sexualidade & Bordini (2014) \\
\hline Gênero, género & Portalino (2017) \\
\hline $\begin{array}{l}\text { Sexualidade e perversão } \\
\text { (psicanálise) }\end{array}$ & Simião (2015) \\
\hline Mulheres Trans & Costa $(2015)$ \\
\hline Pessoas Transsexuais & Baptista (2017) \\
\hline $\begin{array}{l}\text { Saúde de travestis e mulheres } \\
\text { trans; Prostituição trans }\end{array}$ & Miwa (2019) \\
\hline Saúde & Costa (2015) \\
\hline Sujeito soropositivo & David (2018) \\
\hline Violência Sexual contra meninos & Hohendorff (2016) \\
\hline $\begin{array}{l}\text { Violência Sexual contra crianças } \\
\text { e Adolescentes }\end{array}$ & Souza (2014) \\
\hline Violência Doméstica & Rêgo \\
\hline $\begin{array}{l}\text { Violência Sexual contra } \\
\text { Adolescentes }\end{array}$ & Martinho (2016) \\
\hline Diversidad Sexual & Portalino (2017) \\
\hline Aborto & Rebouças (2015) \\
\hline Masturbação Feminina & Baumel (2014) \\
\hline Identidade Sexual & Silva (2017) \\
\hline Abuso sexual & Mozine (2017) \\
\hline Sentimentos sexuais na clínica & Pitanga (2016). \\
\hline Casamento Infantil no Brasil & Pantoja (2019) \\
\hline
\end{tabular}


Um detalhe importante no levantamento bibliográfico é o que denominaremos de "Escalas de Sexualidade Humana", para os estudos e investigações em Educação Sexual. Eles são apresentados no Quadro 3, e incentivam o desenvolvimento de aplicações para fins de estudos quali-quantitativos na área da Educação Sexual.

Quadro 3 - "Escalas da Sexualidade Humana” produzidas no campo da Psicologia para os estudos em Educação Sexual

\begin{tabular}{|c|c|}
\hline ESCALAS & REFERÊNCIAS \\
\hline $\begin{array}{l}\text { Escala de Personalidade de Comrey } \\
\text { (CPS) }\end{array}$ & $\begin{array}{l}\text { Comrey et al. (1965); Comrey } \\
\text { (1970); Rodrigues (1973) }\end{array}$ \\
\hline \multirow{2}{*}{$\begin{array}{l}\text { Escala de Preconceito contra Diver- } \\
\text { sidade Sexual e de Gênero-Revisada }\end{array}$} & Costa et. al. , 2015; 2016 \\
\hline & Pereira et al. (2009) \\
\hline $\begin{array}{l}\text { da Escala do Índice de Função Sexual } \\
\text { Feminina (Female Sexual Function } \\
\text { Index - FSFI) }\end{array}$ & Rosen et al., 2000 \\
\hline $\begin{array}{l}\text { Escala Mosher Abreviada de Culpa } \\
\text { Sexual }\end{array}$ & $\begin{array}{l}\text { Abramson; Mosher, 1975; } \\
\text { Janda; Bazemore, } 2011\end{array}$ \\
\hline $\begin{array}{l}\text { Escala Marlowe-Crowne de } \\
\text { Desejabilidade Social }\end{array}$ & $\begin{array}{c}\text { Evans, 1982; Gouveia et al., } \\
2009\end{array}$ \\
\hline $\begin{array}{l}\text { Escala de Satisfação Sexual para } \\
\text { Mulheres }\end{array}$ & Catão et al., 2010 \\
\hline Escala de Bem-estar Subjetivo (EBES) & $\begin{array}{c}\text { Albuquerque; Tróccoli (2004) } \\
\text { e Pureza et al. (2012). }\end{array}$ \\
\hline
\end{tabular}

Fonte: elaborado pelos autores

Um trabalho de "fôlego" ainda por ser feito, envolve uma Análise Documental (Cellard, 2008), nas disciplinas obrigatórias destes programas, com o intuito de identificar os principais autores(as) trabalhados, os textos desenvolvidos e o modelo das dinâmicas de desenvolvimento destes temas. Isso auxiliaria em muito o trabalho de orientação e o trabalho de investigação de iniciantes à pesquisa, tanto na Psicologia, quanto na Educação, nas licenciaturas, em especial, a Pedagogia.

Ao trabalho de "fôlego" referido anteriormente, soma-se, ainda, o aprofundamento das temáticas, no período, nos recortes de níveis 1 (os 1.439 trabalhos) e 2 (o recorte em 100 trabalhos, distribuídos entre as 
cinco universidades, para fins de leitura dos resumos), que estão na mira dos pesquisadores(as) autores(as) e seus orientandos(as), com intuito de estimular produções, a começar, na iniciação científica de psicólogos(as) e novos e novas pesquisadores(as) na área da Educação Sexual.

$\mathrm{Na}$ ausência de indicação de periódicos no site da CAPES, sob o mesmo descritor composto, a pesquisa não avança em outras bases de dados como Google Acadêmico, por exemplo, por se perceber que, sob este descritor, os trabalhos, quase em sua totalidade, indicam artigos em Educação Sexual, todos voltados à Escola, o que "desencaminha" a problemática e os objetivos da pesquisa de se delimitar a produção em Educação Sexual, estritamente, na área da Psicologia e, portanto, trabalhos produzidos por psicólogos(as) e em Programas de Psicologia.

$\mathrm{Na}$ conclusão se irá apontar os encaminhamentos para a pesquisa em Educação Sexual e algumas contribuições que este estudo traz à Educação, a partir da Psicologia; as ciências psicológicas deixam à disposição da Educação, em especial a Educação Sexual um importantíssimo apontamento sobre práticas em e sobre a sexualidade humana.

\section{CONCLUSÃO}

Que encaminhamentos à pesquisa este texto pôde proporcionar à formação inicial para pesquisadores(as) desde a graduação, em cursos de psicologia e outras áreas afins, como a Educação?

- Encaminhamento 1: na área da Psicologia, sem a consideração de áreas, como a Psicologia Social, Psicologia Escolar e outras denominações correlatas, na particularidade dos Programas de Pós-Graduação em Psicologia brasileiros, as pesquisas em Educação Sexual não se limitam aos espaços escolares e, aliás, nem todas as Linhas dos Programas e as pesquisas localizadas na CAPES são diretamente voltadas aos temas educacionais, o que permite, por outro lado, o acesso destas pesquisas para transposições à Educação ou outras áreas afins; na Educação, particularmente, ao tema do currículo, seja para a educação básica, seja para um currículo de formação de professores(as) em Educação Sexual, inicial e continuada. Neste primeiro encaminhamento: as pesquisas e as instituições que mais produzem conhecimento em Educação Sexual, na Psicologia, efetuam o avançar e a amplitude das temáticas em 
sexualidade humana, englobadas numa visão e numa particularidade, a particularidade das ciências psicológicas.

- Encaminhamento 2: Os Programas de Pós-Graduação pesquisados (os cinco primeiros que mais produzem nos temas da Educação Sexual) não possuem nas denominações das suas respectivas Linhas de Pesquisa o termo "Educação Sexual", mas estão ali seus respectivos tópicos de reflexão, seus temas de estudo e problemáticas, como: violência contra crianças e mulheres - em maior número - gênero, feminilidades, masculinidades, direitos humanos, pessoas trans, etc. Em nosso entendimento, as Linhas fazem grande apreço ao campo das ciências psicológicas de modo amplo, não, especificamente, de cunho escolar, apesar, de estarem presentes, tanto nas Linhas, quanto em algumas ementas de disciplinas, constantes em seus sites, que não foram profundamente tratados, o que se fará em outro produto textual dos autores, mas que ficam como encaminhamentos de produção aos psicólogos(as), pedagogos(as) e demais áreas que confluem para a área da Educação Sexual.

- Encaminhamento 3: Os temas recorrentes nestes cinco programas indicados sugerem três tendências que devem ser investigadas em profundidade, da Iniciação Científica ao stricto sensu; recomendação tanto para psicólogos em formação, quanto de outras áreas, como as licenciaturas (a Pedagogia), quais sejam: como primeira tendência a temática da violência de gênero (violência e abuso sexual a crianças e mulheres) e a própria questão de gênero, de maneira, mais ampla, sempre no viés psíquico; segunda tendência: escalas de classificação e interpretação das sexualidades (algo ausente nas pesquisas em Educação) e, como última tendência, aspectos psíquicos pouco explorados na área da Educação Sexual, especificamente, como masturbação, saúde coletiva, prazer feminino.

Que contribuições, os estudos e pesquisas em Psicologia aqui apresentados podem ser efetivadas no direcionamento para outras áreas, como a Pedagogia, inicialmente, mas, também para outras licenciaturas no tratamento específico para a Educação Sexual? 
- Contribuição 1: a relação de investigação "clínica x sala de aula (educação escolar)". a produção científica apresentada pelos psicólogos(as) em formação de pesquisa, pode se estendida para outras áreas, na medida e na limitação dos aspectos clínicos, específicos à área, bem como de seus respectivos aspectos teóricos, muito específicos; tais elementos podem ser apropriados ao campo da educação e da pedagogia, sobretudo, na máxima: a clínica não é sala de aula e a sala de aula não é clínica. Mesmo assim, podem se dar em interfaces. Muito do que a Psicologia pode nos oferecer, enquanto práticas de intervenção social.

- Contribuição 2: Pesquisas entre as ciências psicológicas e as ciências da educação. A principal contribuição que se quer apontar aqui é a especificidade da da pesquisa em Psicologia. Os pesquisadores(as) da Educação Sexual deverão observar e atentar para estas especificidades e orientarem suas investigações, respeitando estas especificidades, sobretudo, de autores referenciais de metodologias. Não se refere apenas às Escalas de mensuração em sexualidade humana indicadas neste texto, mas, fundamentalmente, os referenciais teóricos das abordagens em Psicologia. Isso ajudará em muito na produção de pesquisas aplicativas em Educação Sexual.

- Contribuição 3: A Educação Sexual é "tentada" a investigar mais questões relativas ao "fora da escola". A produção das ciências psicológicas nos trazem os mesmos temas da Educação Sexual, dos estudos de gênero e sexualidade, de uma forma de ciência da psicologia, que, quase "nunca", conforme nossos achados, não estão nos espaços da escola, aparecem muito pouco. Desta forma, esta terceira contribuição, a construímos como um dizer investigativo para a Educação; que se invista mais em pesquisas em espaços não escolares e "onde" estão demandas mais amplas que as estritamente voltadas para "conhecimentos" sobre sexualidade. No lugar, uma ênfase na subjetividade dos sujeitos, em suas dimensões de vida, de existência, de conflito, de angústia, etc., relativas à sexualidade. 
Espera-se que o presente estudo "incite" e "excite" - como afirma Foucault (1999) -, novas pesquisas em Educação Sexual, em áreas que produzem conhecimento sobre as ciências da sexualidade, tal como se propõe neste texto, explorando a produção de psicólogos e de psicólogas, no campo da Psicologia. Que se evidenciem não apenas as pesquisas, mas seus respectivos pesquisadores(as) e, sobretudo, as inovações temáticas em curso, que trazem, para a Educação Sexual, grandes subsídios para o espaço escolar e universitário, como também para os espaços sociais mais amplos, promovendo, saúde, bem estar e prazer em viver livre e emancipatoriamente a diversidade sexual, a diversidade humana.

\section{REFERÊNCIAS}

ABRAMSON, P. R.; MOSHER, D. L.; Development of a measure of negative attitudes toward masturbation. Journal of Consulting and Clinical Psychology, 43(4), 485-490, 1975. Disponível em: < https://psycnet.apa.org/record/1975-27350-001 >. Acesso em 10 set. 2020.

ALBUQUERQUE, A. S.; TRÓCCOLI, B. T. Desenvolvimento de uma escala de bem-estar subjetivo. Psicologia: Teoria e Pesquisa, 20 (2), 153-164, 2004. Disponível em: < https:// www.scielo.br/pdf/ptp/v20n2/a08v20n2.pdf >. Acesso em: 05 set. 2019.

ANTUNES, M. A. M. A psicologia no Brasil: leitura histórica sobre sua constituição. 5. ed. São Paulo. EDUC, 2014.

ASSUMPÇÃO JUNIOR, F.; ALMEIDA, T. Sexualidade, Cinema e Deficiência. São Paulo: LMP Editora, 2008.

BAPTISTA, G. T. A. Bem-estar de pessoas transexuais: a contribuição da Psicologia Positiva. 2017. 150 f. Dissertação. (Mestrado em Psicologia) - Universidade Federal do Espírito Santo, Vitória (ES), 2017.

BARROSO, C., BRUSCHINI, M. C. Educação sexual: debate aberto. São Paulo: Vozes, 1982.

BARROSO, C.; BRUSCHINI, M. C. (Orgs.). Sexo e juventude: como discutir a sexualidade em casa e na escola. 2. ed. São Paulo: Brasiliense, 1985.

BAUMEL, S. W. Investigando o papel da masturbação na sexualidade da mulher. 2014. 144 f. Dissertação. (Mestrado em Psicologia) - Universidade Federal do Espírito Santo, Vitória (ES), 2014.

BOCK, A.M.B.; FURTADO, O.; TEIXEIRA, M,L. Psicologias: uma introdução ao estudo da psicologia. São Paulo: Saraiva, 2001. 
BORDINI, G. S. As negociações da articulação entre gênero e sexualidade na interação de jovens universitários. 2014. 215 f. Tese. (Doutorado em Psicologia) - Instituto de Psicologia, UNIVERSIDADE FEDERAL DO RIO GRANDE DO SUL, Porto Alegre, 2014.

BORGES, L. S.; CANUTO, A. A. A.; OlIVEIRA, D. P.; VAZ, R. P. (2013). Abordagens de gênero e sexualidade na Psicologia: Revendo conceitos, repensando práticas. Psicologia: Ciência e Profissão, 33(3), 730-745, 2013. Disponível em: < https://doi.org/10.1590/ s1414-98932013000300016 >. Acesso em: 05 set. 2020.

CAMINO, L.; PEREIRA, C. R. O papel da Psicologia na construção dos direitos humanos: análise das teorias e práticas psicológicas na discriminação ao homossexualismo. Perfil, 13(13), 49-69, 2000. Disponível em: < https://doi.org/10.1590/S0102$37722013000100010>$. Acesso em: 05 set. 2020.

CATÃO, E. et al. Escala de Satisfação Sexual para Mulheres: Tradução, adaptação em estudo preliminar com amostra clínica. Boletim de Psicologia, vol. 60, n. 133, pp. 181-190, 2010. Disponível em: < http://pepsic.bvsalud.org/scielo.php?script=sci_ abstract\&pid=S0006-59432010000200005\&lng=pt\&nrm=iso >. Acesso em: 10 set. 2020 .

COMReY, A. L. The Comrey Perwrullity Scale. San Diego, Educational \& Industrial Testing Service, 1970.

COMREY, A. L.; MESCHIERI, L.; MISITI, R.; NENCINI, R. A comparison of personality factor structure in American and Italian subjects. Journal of Personality and Social Psychology, 1 (3), 257-261, 1965. Disponível em: < https://psycnet.apa.org/record/1965-10188-001 >. Acesso em: 05 set. 2019.

COSTA, A. B. Vulnerabilidade para o HIV em mulheres Trans: o papel da psicologia e o acesso à saúde. 2015163 f. tese. (Doutorado em Psicologia) - Universidade Federal do Rio Grande Do Sul, Porto Alegre (RS), 2015.

COSTA, A. B.; BANDEIRA, D. R.; NARDI, H. C. Avaliação do preconceito contra diversidade sexual e de gênero: Construção de um instrumento. Estudos de Psicologia, 32(2), 163 172, 2015. Disponível em: < https://doi.org/10.1590/0103-166X2015000200002 >. Acesso em: 10 set. 2020.

COSTA, A. B.; MACHADO, W. L.; BANDEIRA, D. R.; NARDI, H. C. Validation study of the revised version of the Scale of Prejudice Against Sexual and Gender Diversity in Brazil. Journal of Homosexuality, 63(11), 1446-1463, 2016. Disponível em: < https:// doi.org/10.1080/00918369.2016.1222829 > . Acesso em: 10 set. 2020.

DAVID, G. H. F. Constituição do sujeito soropositivo e seus trajetos de (des) amor. 2017. 251 f. Dissertação. (Mestrado em Psicologia) - Universidade Federal de Juiz de Fora, Juiz de Fora, MG, 2017. 
EKINS, R.; KING, D. The Transgender Phenomenon. London: SAGE, 2006.

EVANS, R. G. Clinical relevance of the Marlowe-Crowne scale: a review and recommendations. Journal of Personality Assessment, 46(4), 415- 425, 1982. Disponível em: <https://www.tandfonline.com/doi/abs/10.1207/s15327752jpa4604_14 >. Acesso em: 10 set. 2020 .

FIGUEIREDO, L.C. Matrizes do pensamento psicológico. Petrópolis: Vozes, 1991.

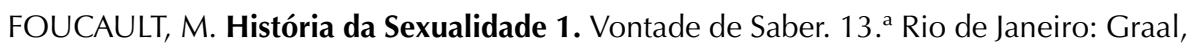
1999.

GADAMER, H. G. Verdade e método II: complementos e índice. Petropólis:Vozes, 2002.

GADAMER, H. G. Hermenêutica em retrospectiva. Petropólis: Vozes, 2012.

GADAMER, H. G. Verdade e método I: traços fundamentais de uma hermenêutica filosófica. 15ª ed. Petropólis:Vozes, 2015.

GOUVEIA, V. V.; GUERRA, V. M.; SOUZA, D. M. F.; SANTOS, W. S.; COSTA, J. M. Escala de desejabilidade social de Marlowe-Crowne: evidências de sua validade fatorial e consistência interna. Avaliação Psicológica, 8(1), 87-98, 2009. Disponível em: < http:// pepsic.bvsalud.org/scielo.php?script=sci_arttext\&pid=S1677-04712009000100008 >. Acesso em: 10 set. 2020.

HOHENDORFF, J. V. Dinâmica da violência sexual contra meninos. 2016. 128 f. Tese. (Doutorado em Psicologia) - Universidade Federal do Rio Grande do Sul, Porto Alegre (RS), 2016.

JANDA, L. H.; BAZEMORE, D. The revised Mosher sex-guilt scale: its psychometric properties and a proposed ten-item version. Journal of Sex Research, 48(4), 392-396, 2011. Disponível em: < https://www.tandfonline.com/doi/abs/10.1080/00224499.2010. $482216>$. Acesso em: 10 set. 2020.

KELLER, F. A definição da Psicologia. São Paulo: E.P.U, 1970.

KUPFER, M.C. Educação para o futuro: psicanálise e educação. São Paulo: Escuta. 2007. MARTINHO, L. O. P. Violência sexual contra adolescentes e sua relação com outras violências. 2016. 72 f. Dissertação. (Mestrado em Psicologia) - Pontifícia Universidade Católica de Goiás, Goiânia, 2016.

MARX, M.H; HILLIX, W.A. Sistemas e teorias em Psicologia. São Paulo: Cultrix, 1976.

MINAYO, M.C.S. Pesquisa Social: teoria, método e criatividade. Petrópolis, Vozes, 1994.

MIWA, H. Y. C. A percepção do cuidado com a saúde de travestis e mulheres transexuais vivenciando a prostituição na cidade de Manaus/AM: análise compreensiva dos discursos. 2019. 90 f. Dissertação. (Mestrado em Psicologia) - Programa de Pós- 
Graduação em Psicologia - Universidade Federal do Amazonas, Manaus, 2019.

MOZINE, A. C. S. Adolescente sempre é vítima, mas, às vezes...: Representações sociais sobre abuso sexual e compreensões de gênero para Conselheiros Tutelares de municípios do ES. 2017, 150 f. Dissertação. (Mestrado em Psicologia) - Universidade Federal do Espírito Santo, Vitória, ES, 2017.

PANTOJA, C. M. F. Malcher. "Para inglês ver": sobre o casamento infantil em Belém do Pará. 2019. 113 f. Dissertação. (Mestrado em Psicologia) - Programa de Pós Graduação em Psicologia - Universidade Federal do Pará, Belém, 2019.

PEREIRA, A. S. L. S.; MONTEIRO, M. B.; CAMINO, L. Estudo da validação das escalas de crenças sobre a natureza da homossexualidade e de preconceito contra homossexuais. Laboratório de Psicologia, 7(1), 21-32, 2009. Disponível em: < https://doi.org/10.14417/ Ip.683 >. Acesso em: 10 set. 2020.

PITANGA, A. V. Conversas sobre sentimentos sexuais na relação terapêutica. 2016. 174 f. Tese. (Doutorado em Psicologia) - Pontifícia Universidade Católica de Goiás, PUCGOIÁS, Goiânia, 2016.

PORTALINO, E. A. Prejuicio contra diversidad sexual y de género en estudiantes universitarios de Lima. 2017. 48 f. Dissertação. (Mestrado em Psicologia) - Universidade Federal do Rio Grande Do Sul, Porto Alegre, RS, 2017.

POTTER, J.; WETHERELL, M. Discourse and social psychology. London: SAGE, 1997.

PUREZA, J. R.; KUHN, C, H. C.; CASTRO, E. K.; LISBOA, C. S. M. (2012). Psicologia positiva no Brasil: uma revisão sistemática da literatura. Revista Brasileira de Terapias Cognitivas, 8(2), 109-117, 2012. Disponível em: < http://pepsic.bvsalud.org/scielo. php?script=sci_abstract\&pid=S1808-56872012000200006\&lng=pt\&nrm=iso >. Acesso em: 08 set. 2020.

REBOUCAS, M. E. S. S. Aborto: um fenômeno sem lugar. Uma experiência de plantão psicológico a mulheres em situação de abortamento. 2015. 197 f. Tese. (Doutorado em Psicologia) - Universidade Federal do Rio Grande Do Norte, Natal (RN), 2015.

RÊGO, S. F. M. (Re)contando histórias: relatos autobiográficos de violência doméstica, abrigadas em Natal/RN. 2016. 148 f. Dissertação. (Mestrado em Psicologia) - Universidade Federal do Rio Grande do Norte, Natal (RN), 2016.

REY, F.L.G. Pesquisa Qualitativa em Psicologia: caminhos e desafios. São Paulo, Pioneira Thomson, 2005.

RODRIGUES, A. Manual para as Escalas de Personalidade de Comrey; manual, caderno de itens, folhas de resposta e folhas de perfil. PUC/RJ, Departamento de Psicologia, 1973. 
ROSEN, R.C.; BROWN, C.; HEIMAN, J.; LEIBLUM, S.; MESTON, C.M.; SHABSIGH, R.; FERGUSON, R.; D'AGOSTINHO JUNIOR, R. The Female Sexual Function Index (FSFI): a multidimensinal self-report instrument for the assessment of female sexual function. J Sex Marital Ther, 26(2)::191-208, Apr-Jun. 2000. Disponível em: < https://pubmed.ncbi.nlm. nih.gov/10782451/ >. Acesso em: 10 set. 2020.

SAMPIERI, R.H.; COLLADO C.F.; LUCIO, M.D.P.B. Metodologia da Pesquisa. Porto Alegre: Artmed, 2006.

SILVA, O. R. M. Trajetórias de vida e mudança de identidade sexual: quando não se encontra o pote de ouro no final do arco-íris. 2017, 121 f. Dissertação. (Mestrado em Psicologia) - Universidade Federal do Espírito Santo, Vitória (ES), 2017.

SIMIAO, A. R. M. Sexualidade e perversão na psiquiatria de krafft-Ebing. 2015. $268 \mathrm{f}$. Dissertação. (Mestrado em Psicologia) - Universidade Federal de Juiz de Fora, Juiz de Fora, MG, 2015.

SOUZA, V. P. Violência sexual intrafamiliar contra crianças e adolescentes: o estado da arte em periódicos em psicologia. 2014. 192 f. Dissertação (Mestrado em Psicologia, Sociedade e Qualidade de Vida) - Universidade Federal do Rio Grande do Norte, Natal, 2014.

Recebido em: 18/11/2020

Aceite em: 01/12/2020 